\title{
Réalités et hypothèses sur la forme de reproduction sexuée du Sporotbrix schenckii
}

\author{
par M. ANSEL et M. THIBAUT \\ Laboratoire de Parasitologie et de Mycologie ( $\mathrm{P}^{r}$ M. LARIVIÈRE) \\ U.E.R. Cordeliers, 15, rue de l'Ecole de Médecine, F. 75006 Paris
}

\begin{abstract}
Résumé
Sporothrix schenckii a été examiné en microscopie photonique ainsi qu'en microscopie électronique à transmission et à balayage. Le stade parfait a été découvert. Les asques ont quatre spores, ils sont formés directement sur le mycélium et il n'y a pas de fructification. Des commentaires détaillés sont rapportés sur cette forme parfaite.
\end{abstract}

\section{Summary}

Sporothrix schenckii was examined using the light microscope, the electron microscope and the scanning microscope. The perfect state was discovered. The asci are 4 spored, they are formed directly upon the mycelium without fructification. Some comments are reported with emphasis on this perfect state.

Nous remercions bien vivement M. le Professeur M. Chadefaud, de la Faculté des Sciences de Paris, pour ses précieux conseils et ses avis autorisés. 
Au cours de ces dix dernières années, maints auteurs travaillant sur des champignons, dont on ne connaissait jusqu'alors que le ou les modes de multiplication végétative, ont recherché et trouvé la forme dite «parfaite» de reproduction sexuée. Cette forme se manifeste le plus souvent par des asques, quelquefois par des basides. Ainsi peut-on assigner à d'anciens Adélomycètes ou «fungi imperfecti», une place dans la classe soit des Ascomycètes, soit des Basidiomycètes.

Jusqu'à présent, le Sporothrix schenckii se classait parmi les champignons Adélomycètes dans le groupe des conidiosporés. Il se distingue en effet des véritables Sporotrichum qui eux forment des aleuries, par le fait qu'il présente des conidies, soit disposées en manchons autour des filaments, soit groupées en bouquets à l'extrémité de ceux-ci. Ces filaments indifférenciés ne constituent pas des «sporophores " au sens strict du terme.

Aussi, ceux qui poursuivaient des re:her:hes sur le Sporothrix schenckii avaient-ils soit l'attention en éveil pour une éventuelle observation, soit l'imagination au travail pour évoquer un rattachement possible à un groupe connu. Il y avait somme toute deux démarches intellectuelles différentes: soit une observation directe dans des conditions offrant des chances de découverte, soit des déductions spéculatives sur des ressemblances avec des formes similaires. Dans le premier cas, on devait avec des souches de Sporothrix confirmées obtenir des formes sexuées. Dans le deuxième cas, on pouvait tenter un rapprochement des formes Sporothrix avec des genres sexués connus d'Ascomycètes.

\section{I. - Formes Sporothrix d'Endomycetaceae.}

Au cours d'un travail important sur le Sporothrix schenckii, l'un de nous avait pu s'assurer des chances nouvelles, d'une part en utilisant la technique des observations en microscopie électronique, d'autre part en se rapprochant des conditions de vie naturelle de ce champignon.

L'observation au microscope électronique à balayage et à transmission à des grandissements allant de 10.000 à 50.000 permettait évidemment de mieux saisir non seulement la forme extérieure, mais encore les structures intérieures d'un champignon très petit. Ses hyphes, en effet, ont une largeur ne dépassant pas souvent $1 \mu$, ses conidies ont en majorité une largeur de $2 \mu$ et une longueur fréquente de $5 \mu$. On pouvait donc concevoir que si des asques devaient avoir une taille semblable, leurs ascospores seraient plus facilement observées à des grandissements incomparablement supérieurs à ceux donnés par le microssope photonique.

D'autre part, dans la nature, le champignon ne bénéficie pas toujours des conditions optima, qui favorisent plutôt la reproduction végétative par les conidies. L'appauvrissement du milieu, les conditions changeantes de température créent souvent des conditions de souffrance qui déclenchent l'apparition soit des formes de résistance comme les chlamydospores, soit des formes d'origine sexuée comme les asques. Ces derniers sont aussi des sortes de chlamydospores, car la paroi de l'asque protège les ascospores qu'il contient. Il est banal de rappeler que tous les vieux mycologues et tous les mycologues expéri- 
mentés cultivaient souvent sur plâtre, ou sur d'autres milieux défavorables, les champignons dont ils désiraient obtenir la forme de reproduction sexuée.

Il faut beaucoup de patience pour examiner de nombreuses préparations afin d'y étudier en particulier des formes de vieillissement, qui devaient permettre à l'un d'entre nous, d'observer une fois, puis à de nouvelles reprises, des asques contenant des ascospores. Aussi, sur les données d'un appareil végétatif simple mais filamenteux et d'asques libres, non enclos dans un périthèce, n'hésitons-nous pas à rattacher la forme sexuée du Sporothrix schenckii à la famille des Endomycetaceae. Sur cette parenté, un des Maîtres français de la Mycologie, devait nous rassurer après coup, en confirmant notre interprétation. A cause des caractères particuliers de l'asque et des ascospores, nous avons pensé qu'il n'était pas possible d'assigner cette forme au genre Endomyces et il nous a paru nécessaire de créer pour la forme sexuée un nouveau genre: Dolichoascus.

A

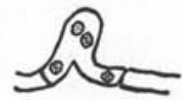

a

B

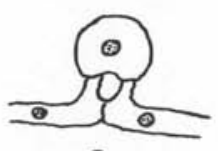

a

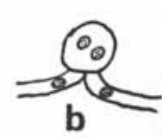

b

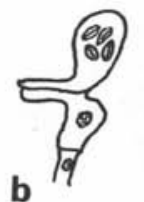

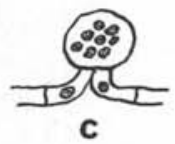

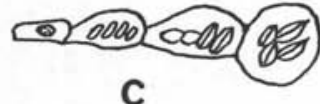

Fig. 1. - A et B. Copulation observée chez des Endomycetaceae A : chez Eremascus fertilis, B : chez Endomycopsis figuliger, C : Formation d'asques par apomixie (d'après Guilliermond)

Pouvait-on interpréter différemment cette forme sexuée? D'une part, nous avions d'abord pensé à l'existence d'endospores, comme en a décrit récemment $\mathrm{H}$. Saez chez les Geotrichum. Néanmoins, nous trouvons surprenant que F. Mariat, qui a cru devoir redécrire plus minutieusement la morphologie de Sporotrichum schenckii (1962) n'ait pas alors signalé des endospores chez un champignon qu'il étudie depuis 1950 . Si pendant ce laps de temps, ses observations ont été alors incomplètes, nous constatons que la découverte d'asques et d'ascospores dans la souche qu'il nous avait aimablement communiquée, l'a incité à rechercher et à signaler enfin des endospores. Mais il faut regretter qu'il n'ait pu donner des photographies objectives de ses préparations, car elles eussent permis d'être assuré qu'il ne s'agit pas d'une fausse interprétation traduite par des dessins. En effet, dans les hyphes âgées, les vacuoles avec leur paroi simple, renferment des substances qui donnent des précipités avec divers colorants et on observe parfois des alignements de vacuoles. Saez a donné des endospores de Geotrichum candidum d'excellentes photographies qui elles, ne laissent aucun doute. En admettant l'existence d'endospores chez Sporothrix schenckii, remarquées enfin après tant d'années d'observations, 
nous trouvons dans les figures données par Mariat un caractère d'endospores commun avec celles décrites par Saez. Ces endospores quand elles sont multiples sont alignées en une seule file dans le sens de l'hyphe. Or dans l'asque de Dolichoascus schenckii, les ascospores décrites par nous, au nombre de quatre théoriquement (trois si la coupe plus fine en laisse une dans un autre plan) sont juxtaposées. La présence banale d'endospores, qui existent en effet et que nous avons nous-même observées, n'empêche nullement un

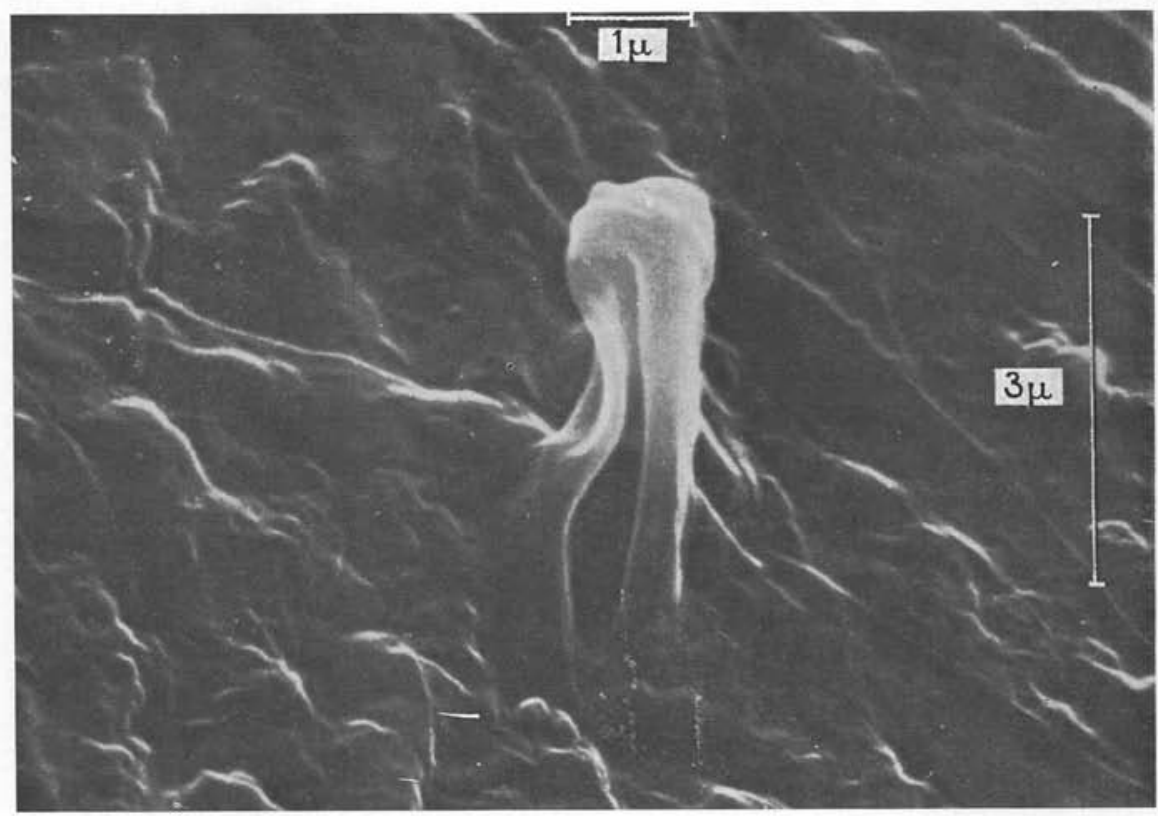

FIG. 2. - Copulation chez 1'Endomycetaceae Dolichoascus schenckii observée au microscope électronique à balayage (d'après Thibaut et Ansel)

champignon de présenter aussi des asques et des ascospores. Dans les figures données par Mariat, deux ou trois images laissent à penser qu'il peut s'agir d'asques. Mais cet auteur n'ayant pas utilisé l'examen en microscopie électronique n'a pu voir des asques et des ascospores, dont il ne pourrait préciser la structure en microscopie photonique, étant donné la très petite taille de ces éléments. Ces asques sont d'ailleurs très peu nombreux. De plus, nous constatons que dans ses figures, Mariat n'a jamais dessiné plus de quatre « endospores » à la file, ce chiffre étant un nombre correspondant à celui d'ascospores, sans que pour autant cela en soit. Mais les incontestables endospores, photographiés par Saez chez Geotrichum candidum sont en file au nombre parfois de 7, 8 et même 13. Enfin, une autre assertion de Mariat nous paraît spécieuse et sujette à caution. Il déclare en effet que dans les cultures âgées où il observe des spores endogènes, 
les conidies exogènes apparaissent en même temps. Mais il ne donne aucune figure de cette curieuse coexistence peu vraisemblable sur le plan physiologique.

$\mathrm{Si}$ après vingt ans d'observations et seulement après notre publication sur les asques du Sporotrichum schenckii, Mariat trouve des endospores, nous pensons que ces endospores ne sont pas si fréquentes et que leur observation n'était guère aisée. L'utilisation de techniques plus modernes permettra sans doute à cet auteur de découvrir quelque jour cette reproduction sexuée qu'il ne peut distinguer à des agrandissements insuffisants.

D'autre part, divers travaux américains, dont tout particulièrement ceux de Carbonell et de Rodriguez au Vénézuela, sur le Paracoccidioïdes brasiliensis, nous avaient

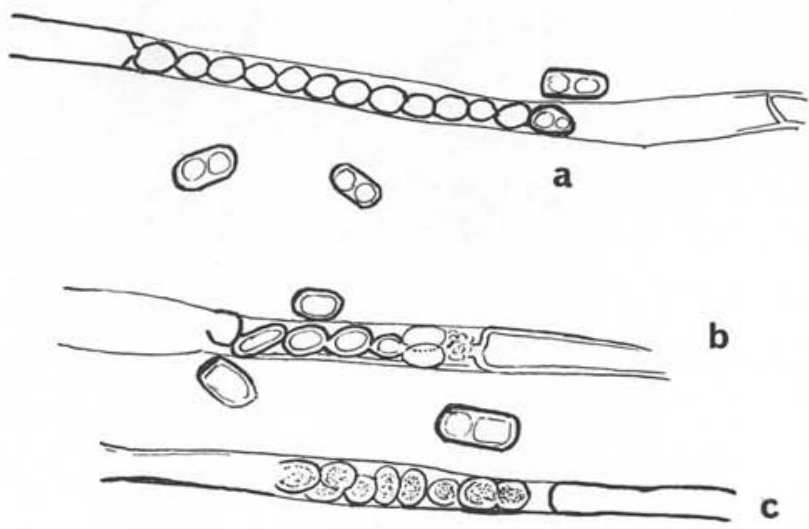

FIG. 3. - Endospores chez Geotrichum candidum a. b. c; (d'après H. Saez)

montré l'existence possible d'hyphes intrahyphales. Lane et Garrison (1970) en ont montré aussi chez Blastomyces dermatitidis et chez Histoplasma capsulatum. Ces hyphes sont apparemment des parasites « cannibales » à l'intérieur d'autres hyphes. Signalons que de telles hyphes bien que pouvant être coupées transversalement, se présentent souvent dans toute leur longueur, que par ailleurs on y reconnaît les structures cytoplasmiques souvent accompagnées de glycogène, de l'hyphe parasitée sénescente ou non ; de plus on n'y observe pas les résidus cellulaires de l'épuisement intra-ascal consécutif à la formation des ascospores. Enfin, sur nos préparations, il nous était possible de voir quatre ascospores immatures côte à côte et même trois absolument mûres sur un même plan. Quel extraordinaire hasard, que celui qui nous aurait pour la première fois montré quatre hyphes ensemble avec des structures anormales et nous les aurait montrées se tassant, comblant l'intérieur d'une autre hyphe entièrement envahie.

Le hasard nous donnait de relire peu de temps après, l'ouvrage d'un grand mycologue du début du siècle, Paul Vuillemin. Or cet auteur considérant un Endomyces crateriformis de Hudelo, Sartory et Monlaur, espèce pathogène découverte dans une lésion 
cutanée chez l'homme, s'apercevait que le mode de reproduction asexuée de ce parasite, plaçait au point de vue végétatif l'Endomyces crateriformis parmi les Sporotrichum (nous dirions aujourd'hui Sporothrix). Aussi nous-même parlant d'un Spcrothrix schenckii

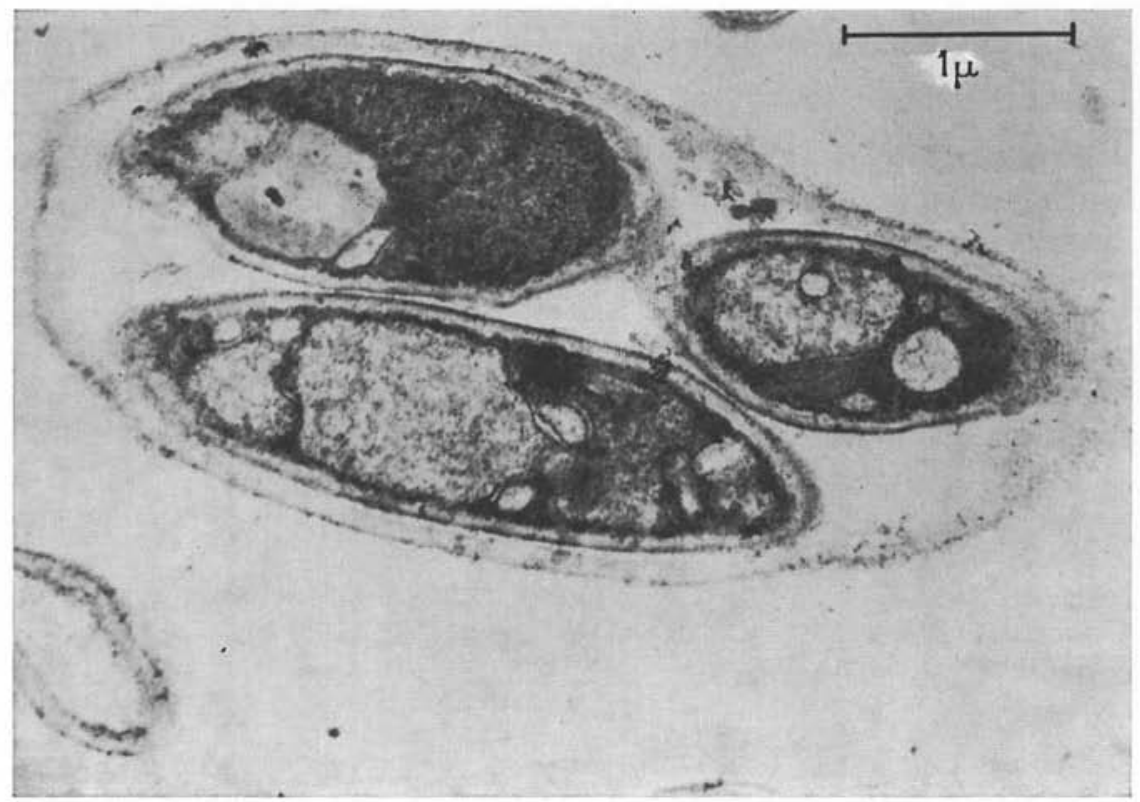

FIG. 4. - L'asque et les ascospores chez Dolichoascus schenckii : microscope électronique à transmission (d'après Thibaut et Ansel)

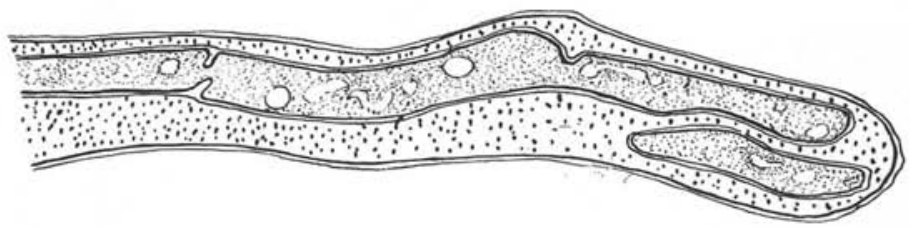

FIG. 5. - Type d'hyphe intra-hytrahyphale chez Paracoccidioïdes brasiliensis (d'après L. M. Carbonell et J. Rodriguez)

typique provenant de la mycothèque de $\mathrm{F}$. Mariat, étions-nous amenés à considérer sa forme sexuée comme une Endomycetaceaz. Vuillemin, par une démarche inverse, était amené à reconnaître comme Sporotrichum la forme végétative de l'Endomyces crateriformis. Voici donc deux Sporothrix pathogènes certains: l'un d'abord connu comme Endomycetaceae se révèle être un Sporotrichum et l'autre d'abord connu comme un Sporothrix se révèle être une Endomycetaceae. 
La conclusion qui s'impose sur le vu de ces formes sexuées, chez des champignons présentant une forme végétative du type Sporothrix, c'est que chez deux espèces, malgré leurs caractères sexuels légèrement différents, il s'agit dans l'un et l'autre cas d'Endomycetaceae: l'un de ces champignons appartient au genre Endomyces: E. crateriforme, l'autre au genre Dolichoascus: D. schenckii. Cependani, il est permis de se demander si d'une part Sporothrix schenckii ne serait pas une espèce collective correspondant à des champignons d'espèces ou même de genres différents, d'autre part si, en poussant plus loin, le Sporothrix schenckii ne pourrait pas être aussi une forme végétative d'un champignon appartenant même à d'autres familles.

\section{II. - Le Sporothrix forme végétative de Ceratocytis?}

Il faut partir de l'observation de faits pour apprézier l'intérêt d'hypothèses, qui, pour ne pas appuyer ce qu'elles voudraient prouver, apportent cependant une nouvelle explication de faits apparemment opposés.

En dehors des Adelomycètes (: Deutéromycètes: Fungi imperfecti), les Ascomycètes comme les Basidiomycètes se reproduisent aussi par des modes divers de multiplication végétative, soit par un seul type, soit par plusieurs types. Ce dernier cas est celui, par exemple, des Ceratocystis sur lesquels nous allons nous étendre plus longuement. Inversement, un même type de reproduction végétative, comme le type Penicillium par exemple, se retrouve chez divers genres ou même dans des familles différentes.

Dès 1928, notre Maître Langeron écrivait à propos des Sporothrix : «Etant donné les aspects polymorphes que nous venons de décrire et leur faculté de donner des conidies-levures, on peut espérer, dans un avenir plus ou moins éloigné, pouvoir le rattacher très probablement à des Ascomycètes, peut-être à des Sphaeriaceae. Chez divers champignons de ce dernier groupe, on a décrit des appareils conidiens qui se rapprochent beaucoup des Rhinocladium 》 (nom de genre auquel Langeron rattachait le Sporothrix).

Aussi n'est-il pas étonnant que dans une autre famille, assez éloignée, celle des Ophiostomataceae, F. Mariat ait trouvé aussi chez le genre Ceratocystis des formes végétatives du type Sporothrix, mais il n'a jamais pu obtenir la transformation du $S p$. schenckii en Ceratocystis. Si ses travaux abondants sur le Sporothrix schenckii ne lui ont pas permis d'y observer les formes sexuées Endomycetaceae, que nous avons trouvé sur une de ses propres souches, c'est pour deux raisons inverses de celles qui nous les ont fait découvrir: d'une part, Mariat n'avait pas utilisé l'examen en microscopie électronique, d'autre part et surtout, les excellents travaux faits par lui sur la physiologie du Sporothrix l'ont amené à cultiver celui-ci sur des milieux riches. Or, sur ces milieux riches en vitamines comme en oligo-éléments, les souches de champignons pathogènes se complaisent dans la reproduction végétative ou présentent, comme le signale d'ailleurs Mariat, des variations morphologiques ou évoluent même vers le pléomorphisme. Cet auteur a pressenti un rapprochement entre le Sporothrix et le Ceratocystis, genre dans lequel la forme végétative peut être du type Verticicladiella. Ces formes, se présentent avec toute la richesse et la beauté de leurs ramifications dans les conditions naturelles de parasitisme sur les Pinaceae et les Taxodiaceae, familles dans lesquelles elles 
déterminent la maladie du bois bleu; mais elles deviennent rabougries, maigres, leurs ramifications sont déformées dès une première culture sur milieu artificiel. Ainsi, la culture sur milieux riches ne permet plus pour ce genre, d'obtenir non seulement des formes sexuées, mais pas même des formes végétatives normales du type Verticicladiella. Que dans ces conditions de culture, un Ceratocystis puisse donner des formes Sporothrix n'a rien d'étonnant. C'est d'ailleurs un fait banal qu'un champignon qui a des for-

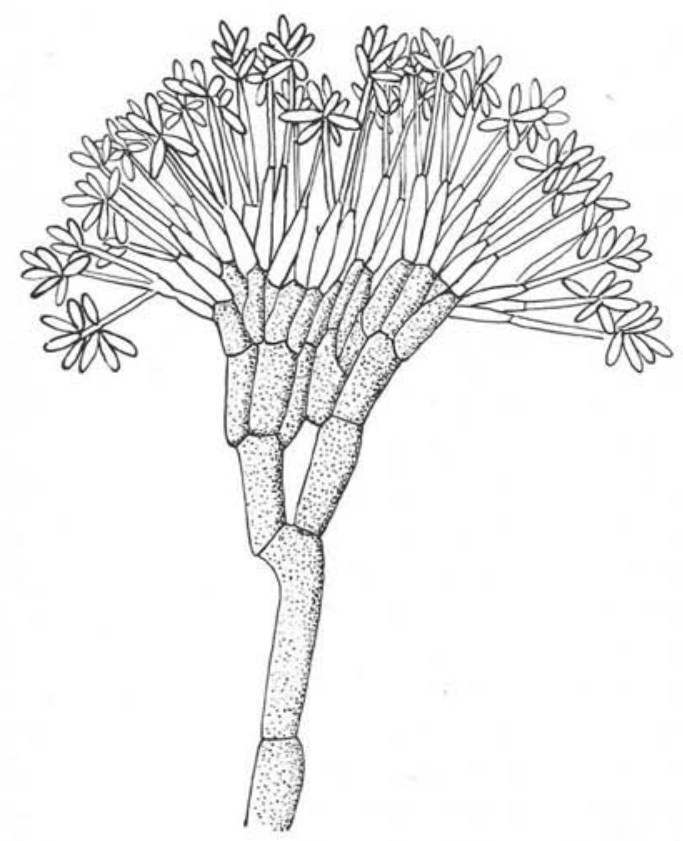

FIG. 6. - Forme verticicladiella d'un Ceratocystis: Vert. penicillata (d'après Kendric)

mes végétatives, considérées comme plus différenciées, plus évoluées, peut toujours donner des formes plus simples.

Toutefois, il ne faut pas voir une raison de rapprochement avec le Sporothrix, parce que le Ceratocystis donne des formes levures. En effet, bien des champignons, qui n'ont avec le Sporothrix schenckii aucune parenté, donnent des formes levures: les Candida qui pourtant filamentent, les Histoplasma qui ont de plus des filaments avec des aleuries, des Mucoraceae comme celle utilisée pour la fermentation de l'alcool de riz.

Ce n'est pas non plus une raison de rapprochement que la constatation dans les lésions de corps astéroïdes. Langeron a obtenu des corpss astéroïdes avec les Actinomyces, les Staphylocoques, le Mycobacterium tuberculosis, le Coccidiö̈des, les Aspergil- 
laceae et même des œufs de bilharzies et aussi des métaux comme le Vanadium (fig. 8). Présenter le corps astéroïde de Ceratocystis comme un indice de parenté avec Sporothrix n'a aucune valeur. Les massues des sphérules astéroïdes ne sont pas d'origine fungique comme le prouve leur non colorabilité par le réactif de Schiff, mais peuvent être interprétées comme une réaction de défense de l'hôte. Récemment, deux auteurs américains donnaient aussi une figure de corps astéroïde chez Histoplasma capsulatum accompagnée du commentaire suivant : «Asteroïd bodies are non specific cell responses to a variety of foreign bodies including yeast cells » (Vanek et Schwarz 1971). Le corps astéroïde est donc une réaction fréquente et même banale du sujet parasité.

FIG. 7. - Conidiophore de type Verticicladiella atrophié chez le Ceratocystis huntii d'après Robinson Jeffrey and Grinchenko

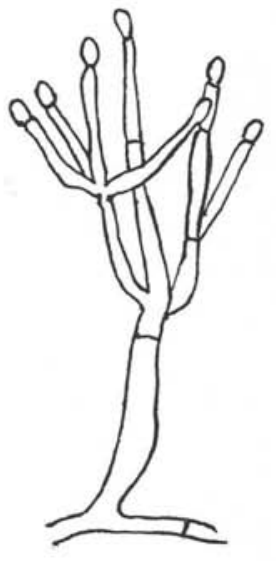

La nature des lésions pathologiques provoquées par des Ceratocystis n'est pas non plus un élément à retenir en faveur d'une éventuelle parenté avec le Sporothrix schenckii. Il est connu qu'un même type de lésion peut être déterminé par des champignons différents, et qu'un même champignon peut provoquer des lésions des types les plus variés.

Quand on écrit «l'élément initial de la formation des perithèces chez les Ceratocystis étudiés est une boucle mycélienne de une ou plusieurs spires. Or une formation exactement comparable est observée chez certaines souches de Sporotrichum schenckii pathogènes », c'est laisser croire à ceux qui l'ignoreraient que des boucles mycéliennes ne se rencontrent que chez les Ceratocystis et les Sporotrichum alors qu'on en voit chez des centaines de genres de champignons, soit à l'origine de leurs perithèces (par exemple chez les Eurotiaceae), soit comme simples éléments apparemment d'ornementation (par exemple chez les Ctenomyces) ou comme éléments d'accrochage.

Lorsqu'on invoque le biotope en écrivant «le biotope de ces champignons est similaire, sinon identique: les Ceratocystis sont des hôtes ou des parasites du bois; $S$. schenckii hôte du sol et des débris végétaux se rencontre fréquemment sur le bois de plantes vivantes comme sur le bois de charpente sain », alors pourquoi n'existerait-il 
Tableau de quelques champignons attaquant le bois

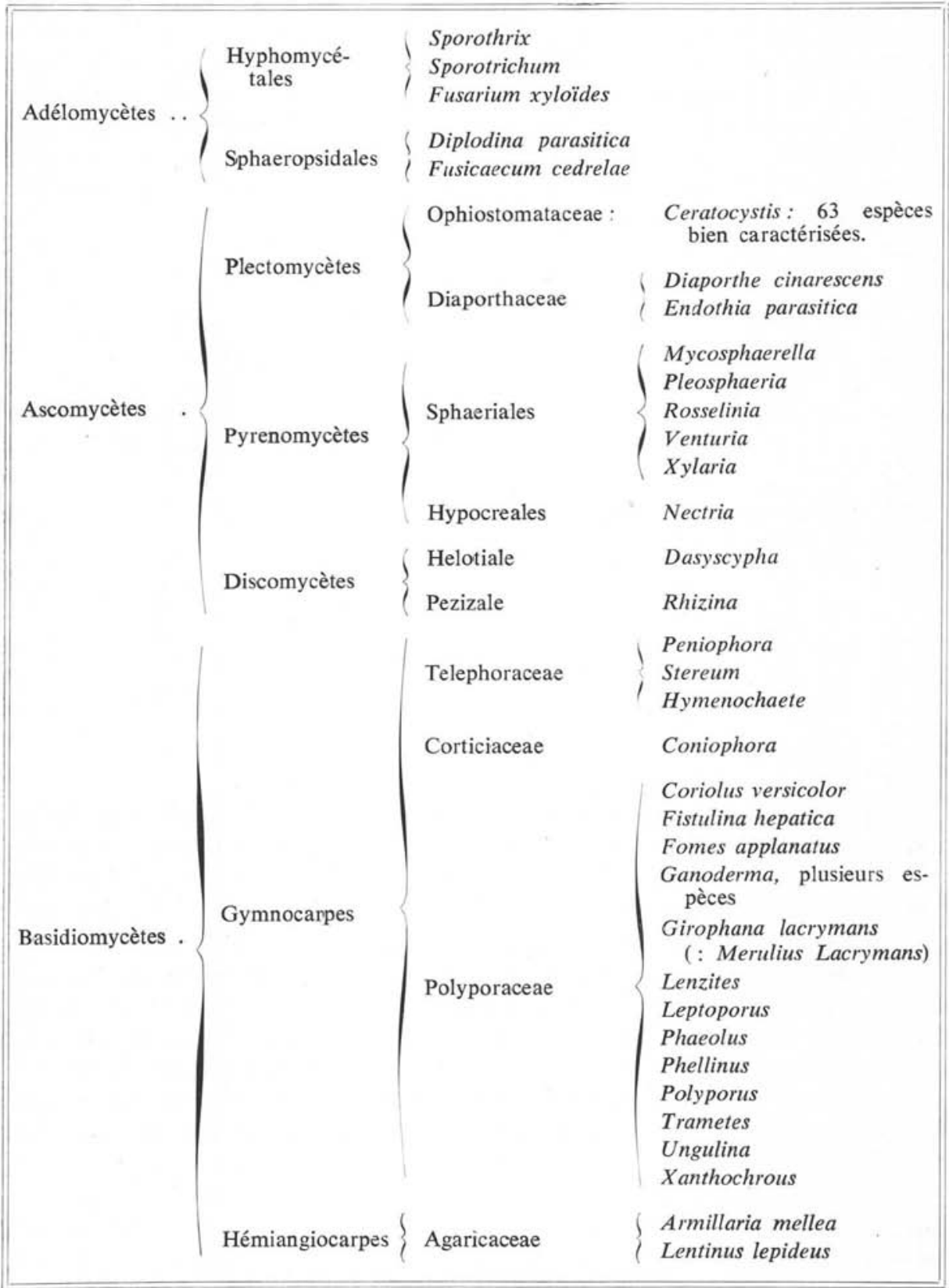

Pour certains genres, nous n'avons pas indiqué leurs trop nombreuses espèces. 


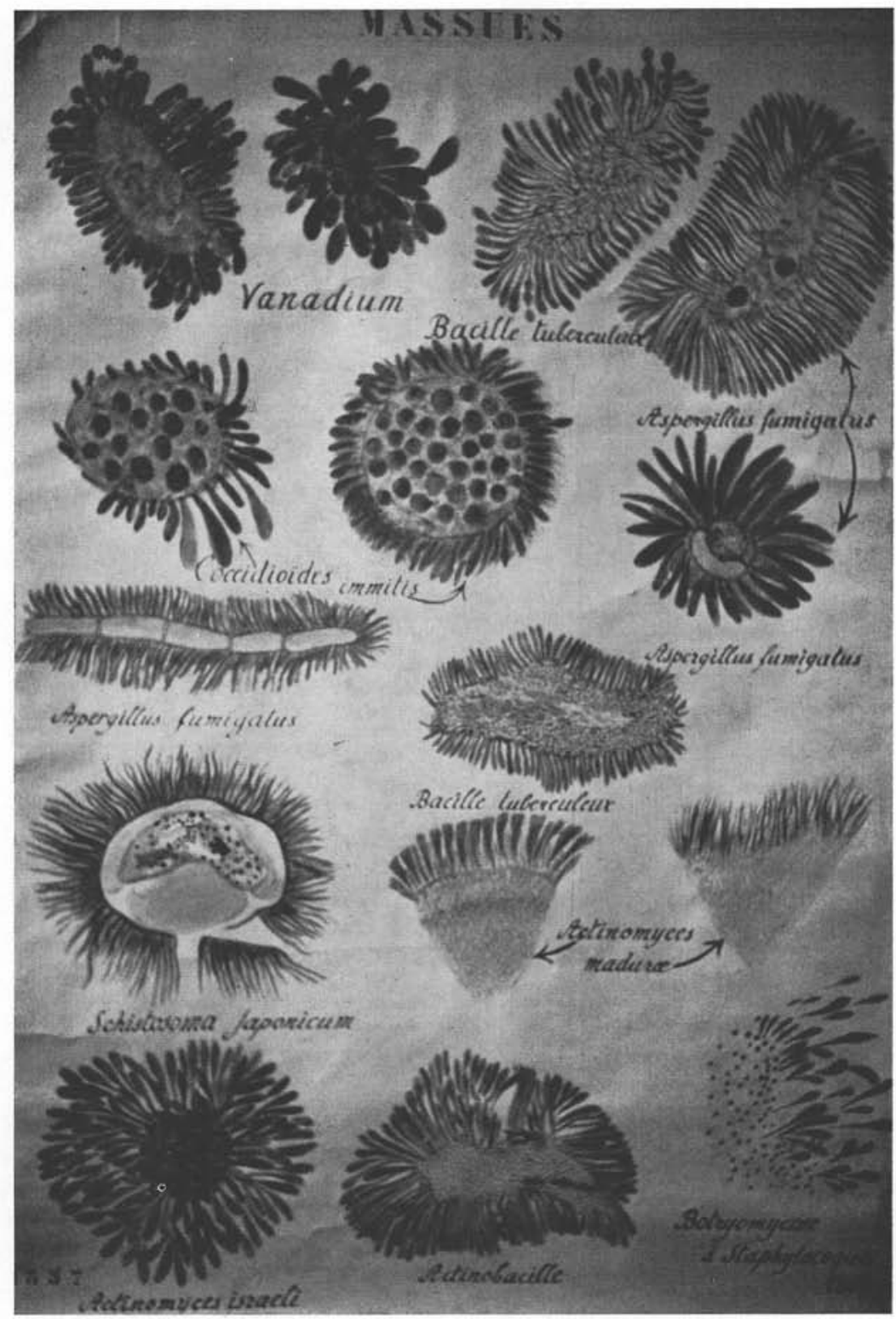

Fig. 8. - Formes massues obtenues avec des agents divers. Minéraux: (Vanadium); Bactériens: (Staphylococcus, Actinobacillus, Mycobacterium, Actinomyces, Streptomyces madurae); fongiques (Coccidioüdes, Aspergillus) ou animaux (Schistosoma) Tableau de $\mathbf{M}$. Langeron 
pas aussi une parenté entre Sporothrix et Merulius lacrymans et les milliers d'espèces parasites de bois morts ou vivants et appartenant aux genres, familles, classes les plus diverses? (cf. tableau).

Enfin, Hunt qui a publié une monographie exhaustive du genre Ceratocystis a bien établi la validité de 63 espèces. Il est regrettable que l'espèce stenoceras à laquelle Mariat rattache sa souche soit précisément exclue de cette longue liste et rangée parmi les espèces insuffisamment connues.

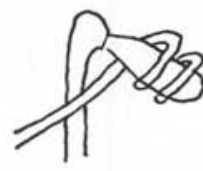

a

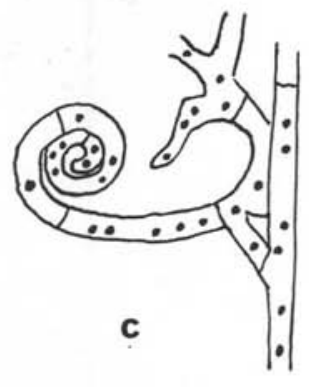

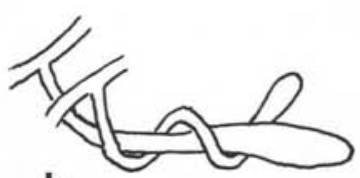

b

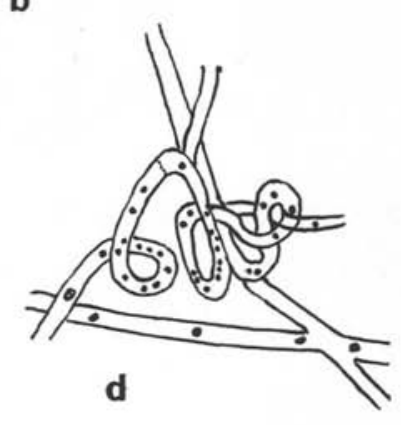

FIG. 9. - Boucles et vrilles. Exemples chez divers Ascomycètes. a) in: Arachniotus trisporus Hots (Gymnoascaceac) d'après Rosenbaum; b) in : Penicilliun vermiculatum (Aspergillaceae) d'après Dangeard; c) in: Sordaria fimicola (Sordariacea) d'après Greis ; d) in: Neurospora sitophila (Melanosporaceae) d'après Schonfeldt

\section{III. - Peut-on concilier ces observations qui sembleraient s'opposer ?}

Si Mariat a pu observer avec un Ceratocystis dont l'espèce reste à confirmer, des formes végétatives de type Sporothrix, il ne signale pas avoir obtenu les formes végétatives de type Verticicladiella ou Leptographium (stricto sensu), ou la forme Chalara, qui caractérisent aussi ce genre. Or cette observation a cependant déjà été faite.

Mais comme nous l'avons fait pour appuyer notre découverte d'une forme sexuée de type Endomycetaceae, nous ferons encore appel aux observations remarquables de ce grand mycologue que fut Vuillemin. Une page de dessins de Vuillemin illustre sa description du Sporotrichum lesnei. Il nomme d'ailleurs celui-ci Rhinocladium lesnei, comme il nomme aussi Rhinocladium schenckii le Sporothrix schenckii. Ce dessin montre à 
l'évidence que l'espèce lesnei en 1 de sa figure 44 présente la forme Sporothrix, mais présente en 7 et 8 la forme Leptographium et même Verticicladiella. Nous retrouvons ce type simplifié de Verticicladiella dans la figure 2 que Robinson Jeffrey et Hutha Grichenko donnent de leur Ceratocystis huntii.

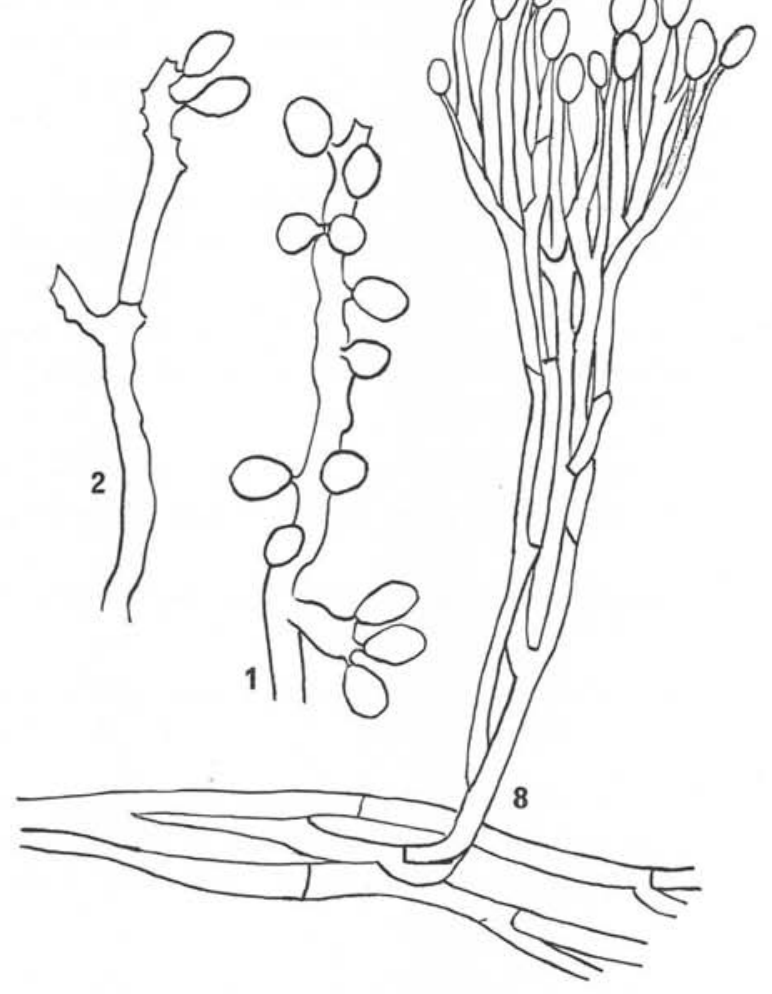

Fig. 10. - Formes vógétatives du type Sporothrix (1 et 2) et Verticicladiella (8) chez le Rhinocladuim Lesnei Vuill. 1910 - d'après Vuillemin 1931

Ainsi, nous paraît parfaitement plausible le fait que des Ceratocystis se présentant aussi sous forme Verticicladiella ou Leptographium (stricto sensu) puissent présenter une forme Sporothrix. Sur le plan pathologique, il est possible qu'un Ceratocystis avec forme Sporothrix, même sans forme Verticicladiella, puisse devenir pathogène car inversement le Sporotrichum lesnei avec sa forme Verticicladiella était bien pathogène puisque «provenant du pied d'un Malgache atteint de mycétome». 
Nous pouvons résumer cet ensemble de faits de la façon suivante. Certaines formes Sporothrix présentent une reproduction de type Endomycetaceae: Sporotrichum crateriforme (Hudelo, Sartory, Montlaur) Vuillemin 1931 est la forme conidienne de l'Endomyces crateriformis de Hudelo, et Sporothrix schenckii (souche 29 Mariat) présente aussi une forme sexuée de type Endonycetaceae: le Dolichoascus schenckii Thibaut et Ansel 1970. Mais, par ailleurs d'autres formes Sporothrix s'apparentent, elles, aux Ophiostomatazeae: le Rhinocladium lesnei pathogène qui est un Sporothrix présente les formes Verticicladiella des Ceratocystis, de même qu'inversement le Ceratocystis stenoceras trouvé par Mariat se présente aussi sous forme Sporothrix.

\section{Conclusion.}

$1^{\circ}$ On a trop précipitamment ramené à la seule espèce $S p$. Schenckii beaucoup d'espèces qui, d'ailleurs, avaient été décrites sans aucun doute à juste raison, comme des espèces différentes.

$2^{\circ}$ Une souche de l'espèce Sporothrix schenckii isolée dans les formes classiques de sporotrichose se rattache au Dolichoascus schenckii, tel que nous l'avons découvert dans la souche 29 que nous a offerte F. Mariat.

$3^{\circ}$ Différents Sporothrix peuvent être:

- soit des Endomycetaceae du genre Endomyces, par exemple Endomyces crateriforme,

- soit des Ophiostomataceae du genre Ceratocystis, par exemple Ceratocystis stenoceras.

$4^{\circ}$ A ces différentes espèces du genre Sporothrix peuvent correspondre des infections tantôt de type différent, tantôt de même nature.

Nous concluerons en disant, du genre Sporothrix, ce qu'écrivait Vuillemin des Sporotrichés. Il forme « un groupe auxiliaire où l'on range les espèces dont la reproduction (sexuée) est inconnue ou trop rare, pour répondre aux besoins urgents de la pratique médicale ».

\section{Bibliographie}

ANSEL (M.), 1957. - Mycoses et champignons parasites de l'homme. Doin éd.

Ansel d'Imeux (M.), 1963. - «Sporotrichose », Encyclopédie Médico-Chirurgicale. Maladies infectieuses, 8124 A 10.

Ansel (M.) et Thibaut (M.), 1970. - Une nouvelle Endomycetaceae: Dolichoascus nov. gen. Découverte de la reproduction sexuée par asques chez le Sporotrichum schenckii (Hetkoen et Perkins, 1900). C.R. Acad. Sciences, 270, 2171-2173.

Carmichael (J.W.), 1962. - «Chrysosporium and some aleuriosporic hyphomycetes». Can. J. of Botany, 40, 1137-1173. 
Carbonell (L. M.) et Rodriguez (J.), 1968. - - Mycelial phase of Paracoccidioïdes brasiliensis and Blastomyces dermatitidis: an electron microscope study». J. of Bacteriol., 96, 533-543.

Fontoynont (M.) et Carougeav, 1922. - « Abcès sous dermiques et gommes ulcérées produits par le Sporotrichum Carougeaui associé à la tuberculose chez un enfant malgache» Bull. Soc. Path. Exot., 15, 444.

Hudelo, Sartory et MONTlaur, 1920. - «Endomyces crateriformis» cité par Vuillemin, p. 1930 in Les champignons parasites et les mycoses de l'homme, Lechevalier éd., p. $69-70$.

Hunr (J.), 1956. - « Taxonomy of the genus Ceratocystis ». Lloydia, 19, 1-59.

Kendrick (W. B.) et Molnar (A.C.), 1965. - «A new Ceratocystis and its Verticicladiella imperfect state associated with the bark beetle Dryocoetes confusus on Abies lasiocarpa ». Can. J. of Bot., 43, 39-43.

LANE (J.W.) et Garrison (R. G.), 1970. - «Electron microscopy of self parasitism by Histoplasma capsulatum and Blastomyces dermatitidis». Mycopathol. Mycol. appl., 40, 271-76.

LANGeron (M.), 1922. - «Un nouveau Sporotrichum malgache: Sporotrichum carougeaui n. sp. ». Bull. Soc. Path. Exot., 15, 453.

—, 1928. - «Mycétomes, Oosporoses, Sporotrichoses, Blastomycoses» in Nouveau traité de Médecine, Masson, éd.

—, 1945. - Précis de Mycologie, Masson, éd.

Mariat (F.), Lavalle (P.) et Destombes (P.), 1962. - « Recherches sur la sporotrichose ». Sabouraudia, 2, 60-79.

Mariat (F.), Escudié (A.) et Gaxotte (P.), 1968. - « Isolement de souches de Ceratocystis sp. à forme conidienne Sporotrichum, de cuirs chevelus humains et de poils de rats. Comparaison avec l'espèce pathogène Sporotrichum schenckii ». C.R. Acad. Sciences, 267, 974-976.

—, 1969. - «Problèmes à propos de l'écologie de Sporotrichum schenckii ». Bull. Soc. fr. Mycol. méd., 15, 11-12.

—, 1969. - « Variant non sexué de Ceratocystis sp. pathogène pour le hamster et comparable à Sporothrix schenckii ». C.R. Acad. Sciences, 269, 2329-2331.

—, Destombes (P.), Diez (E.) et NazimofF (O.), 1970. — « Pouvoir pathogène expérimental chez le hamster et la souris d'une souche de Ceratocystis», Bull. Soc. fr. Mycol. méd., 17, 6-7.

— et Diez (E.), 1971. - «Isolement en Corse de souches de Sporothrix et de Ceratocystis sp. expérimentalement pathogènes pour l'animal ». Bull. Soc. fr. Mycol. méd., 19, 26-27.

—, —, 1971. — « Nature des spores endogènes de Sporothrix schenckii, (Hetkoen et Perkins). Remarques à propos de l'éventuelle forme sexuée de ce champignon », C.R. Acad. Sci, 272, 1075-1077.

Robinson-JefFrey (R. C.) et Grinchenko (A. H. H.), 1964. - «A new fungus in the genus Ceratocystis occuring on blue stained lodgepole pine attacked by bark beetles ». Can. J. of Bot., 42, 527-532. 
SAËZ (H.), 1969. - «Formation d'endospores chez Geotrichum candidum». Ann. Parasit. hum. et comp., 44, 197-204.

Thibaut (M.), 1970. - «Etude biologique, morphologique, cytologique et taxinomique du Sporotrichum schenckii (Hetkoen et Perkins) de Beurmann et Gougerot: Dolichoascus schenckii ( $\mathrm{H}$. et $\mathrm{P}$.), Thibaut et Ansel, 1970 ». Thèse de Doctorat èsSciences, Paris.

—, 1971. - « Recherches en microscopie à balayage sur la morphologie du Sporotrichum schenckii (Hetkoen et Perkins, 1900). Ann. Parasit. hum. et comp., 46, 321-327.

- et ANSEl (M.), 1970. - « Premières observations sur le Dolichoascus schenckii, forme sexuée du Sporotrichum schenckii" ». Bull. Soc. fr. Dermatol.

Victoria (R.-V.), DE BièVRe (C.) et Mariat (F.), 1970. — « Résultats préliminaires concernant la parenté chimique entre Ceratocystis sp. G 118 et Sporothrix schenckii IP 29 ». Bull. Soc. fr. Mycol. méd., 17, 7-8.

Vuillemin (P.), 1931. - Les champignons parasites et les mycoses de l'homme, Lechevalier, éd., Paris. 